\title{
Custo da instalação e desligamento de hemodiálise em pacientes com cateter venoso central
}

\author{
Cost of installing and turning off hemodialysis on patients with central venous catheters
}

Costo de instalación y desconexión de hemodiálisis en pacientes con catéter venoso central

Gillene Santos Ferreira ${ }^{1}$, Monique Christine de Aguiar ${ }^{2}$, Antônio Fernandes Costa Lima ${ }^{3}$

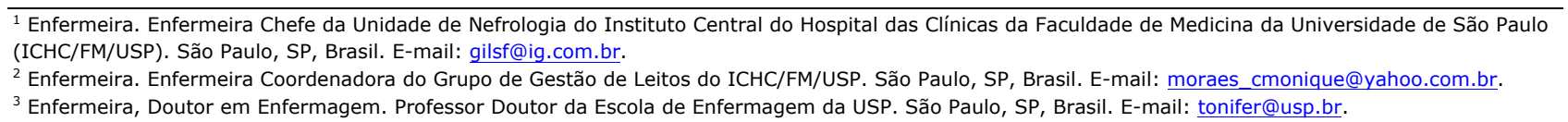

${ }^{3}$ Enfermeira, Doutor em Enfermagem. Professor Doutor da Escola de Enfermagem da USP. São Paulo, SP, Brasil. E-mail: tonifer@usp.br.

\section{RESUMO}

Objetivou-se identificar o custo total médio (CTM) da instalação e desligamento de hemodiálise em pacientes portadores de cateter venoso central. Trata-se de pesquisa quantitativa, exploratória e descritiva na modalidade estudo de caso único, realizada em um hospital universitário público. A amostra, não probabilística, correspondeu à observação de cem instalações e cem desligamentos de hemodiálises em 42 pacientes, durante 23 dias de coleta. Obteve-se o CTM multiplicando-se o tempo despendido por profissionais de enfermagem pelo custo unitário da mão de obra direta, somando-se ao custo dos materiais, soluções e medicamentos. Para a realização dos cálculos, utilizou-se a moeda brasileira ( $R \$$ ). O CTM da instalação foi de $R \$ 80,10$; e do desligamento, de $R \$ 13,04$, totalizando $R \$ 93,14$ por sessão de hemodiálise. Os resultados obtidos favorecerão um melhor planejamento da alocação de recursos humanos, materiais e financeiros, assim, propiciando o incremento de estratégias gerenciais visando à eficiência econômica.

Descritores: Enfermagem; Diálise Renal; Custos Diretos de Serviços; Custos e Análise de Custo; Controle de Custos.

\section{ABSTRACT}

The objective was to identify the average total cost (ATC) for installing and turning off hemodialysis on patients with central venous catheters. This quantitative, exploratory, and descriptive research, in the mode of a single-case study, was conducted in a public university hospital. The non-probabilistic sample corresponded to the observation of 100 installations and 100 terminations of hemodialysis on 42 patients during 23 days of collection. The ATC was calculated by multiplying the time spent by nurses by the unit cost of direct labor, and adding the cost of materials, solutions, and medications. The Brazilian currency $(R \$)$ was used for the calculations. The ATC for installation was $R \$ 80.10$ and for shutting off was $R \$ 13.04$, totaling $R \$ 93.14$ per hemodialysis session. The results obtained will facilitate a better planning of the allocation of human, material, and financial resources enabling the increase of managerial strategies aimed at economic efficiency.

Descriptors: Nursing; Renal Dialysis; Direct Service Costs; Costs and Cost Analysis; Cost Control.

\section{RESUMEN}

Se objetivó identificar el costo medio total (CMT) de la instalación y desconexión de hemodiálisis en pacientes portadores de catéter venoso central. Investigación cuantitativa, exploratoria, descriptiva, modalidad estudio de caso único, realizada en hospital universitario público. Muestra no probabilística correspondiente a la observación de 100 instalaciones y 100 desconexiones de hemodiálisis en 42 pacientes durante 23 días de recolección. Se obtuvo el CMT multiplicando el tiempo empleado por profesionales por el costo unitario de mano de obra directa, sumándosele el costo de materiales, soluciones y medicamentos. Para la realización de los cálculos, se utilizó moneda brasileña ( $\$$ ). El CMT de la instalación fue de $R \$ 80,10$, y el de la desconexión, $R \$ 13,04$; totalizando $R \$ 93,14$ por sesión de hemodiálisis. Los resultados obtenidos favorecerán una mejor planificación de la utilización de recursos humanos, materiales y financieros, haciendo propicio el incremento de estrategias gerenciales apuntadas a la eficiencia económica.

Descriptores: Enfermería; Diálisis Renal; Costos Directos de Servicios; Costos y Análisis de Costo; Control de Costos. 


\section{INTRODUÇÃO}

Nos últimos 10 anos a taxa de prevalência estimada de pacientes com doença renal (DR), que necessitam de diálise para sobreviver, praticamente dobrou e mantémse em uma crescente mostrando que, anualmente, aumenta a quantidade de pessoas dependente de Terapias Renais Substitutivas (TRS). As TRS constituemse em tratamentos de alto custo e, por isso, tem gerado grande preocupação econômica para o Sistema Único de Saúde (SUS) ${ }^{(1)}$.

O grande volume de recursos financeiros do orçamento ambulatorial do SUS destinado aos procedimentos relativos às TRS e a perspectiva da tendência de aumento exponencial desta alocação, decorrente do aumento da expectativa de vida de portadores de DR, configuram a avaliação econômica como importante aliada para subsidiar a alocação exequível e eficiente de recursos ${ }^{(2)}$.

A hemodiálise (Hd) é a modalidade de TRS mais utilizada para portadores de DR portanto há a imprescindibilidade da produção de conhecimento que permita relacionar o uso de materiais específicos, soluções/medicamentos, mão de obra especializada e os custos para sua viabilidade ${ }^{(3)}$.

A execução da Hd requer a observação de requisitos básicos, como a obtenção de uma via de acesso à circulação sanguínea, utilização de soluções, materiais e equipamentos específicos, além da disponibilidade de recursos humanos e estruturais que contemplem as Resoluções estabelecidas pelo Ministério da Saúde $(\mathrm{MS})^{(4)}$.

Atualmente, existem cerca de 590 centros especializados ou clínicas de $\mathrm{Hd}$ em todo o cenário nacional ${ }^{(5)}$. O repasse monetário do SUS às unidades hospitalares, cadastradas e referenciadas pelo MS por meio do Sistema de Gerenciamento de Tabela de Procedimentos, Medicamentos, Órteses e Próteses e Materiais Especiais (SIGTAP), referente à Hd destinada à pacientes renais agudos/crônicos agudizados, sem tratamento dialítico iniciado, corresponde a $\mathrm{R} \$ 111,42$. De acordo com o SIGTAP esta modalidade de TRS, considerada de média complexidade, cobre idades de zero a 110 anos, sendo o tipo de financiamento de média e alta complexidade ${ }^{(6)}$.

Tendo em vista que a Hd é a modalidade de TRS mais utilizada para portadores de DR, bem como a imprescindibilidade da produção de conhecimento que permita relacionar o uso de materiais específicos, soluções/medicamentos, mão de obra especializada e os custos para sua consecução, realizou-se esta pesquisa.
Destarte, objetivou-se identificar o custo total médio (CTM) da instalação e do desligamento de $\mathrm{Hd}$ em pacientes portadores de cateter venoso central (CVC), atendidos em um hospital de ensino e pesquisa, público, de grande porte.

\section{MÉTODOS}

Trata-se de pesquisa quantitativa, exploratória, descritiva, na modalidade de estudo de caso único ${ }^{(7)}$. Foi realizada na sala de $\mathrm{Hd}$, localizada no 70 andar do Instituto Central (IC) do Hospital das Clínicas da Faculdade de Medicina da Universidade de São Paulo (HC-FMUSP), São Paulo-SP, destinada ao atendimento de, em média mensal, 17 pacientes renais agudos e crônicos

Uma equipe composta por médicos nefrologistas, assistentes e residentes, realiza interconsultas no Hospital, priorizando o IC, e indica a modalidade terapêutica a ser executada junto aos pacientes em urgência dialítica atendidos nesta sala.

A equipe de enfermagem da sala é composta por um enfermeiro e dois auxiliares por turno (manhã, tarde, noite) que trabalham seis horas/dia ou 12 horas/dia, conforme escala mensal.

Todo o material utilizado, como exemplos, dialisadores capilar, linhas arterial e venosa (circuito extracorpóreo), isoladores, seringas, agulhas e soluções/medicamentos, tais como, galões de bicarbonato de sódio 8,4\% com água para injeção, solução polietrolítica ácidos e básicos, bolsas de soros, manitol, heparina são armazenados em locais específicos na própria sala.

O circuito extracorpóreo e o isolador não são reprocessados, pois os pacientes atendidos não possuem sorologias para HIV, Hepatite B e Hepatite C conhecidas, em tempo hábil, uma vez que são internados no complexo HC-FMUSP em urgência dialítica.

A pesquisa foi aprovada pela Comissão de Ética e Pesquisa do HC-FMUSP (CAAE: 03825512.8.0000.0068, parecer número 35847). Assegurou-se, por meio da apresentação e da assinatura do Termo de Consentimento Livre e Esclarecido, a cada participante, o anonimato e a garantia de que os resultados obtidos seriam utilizados e divulgados com finalidade única de contribuir para o conhecimento científico, sem qualquer ganho pessoal ou econômico para os pesquisadores.

A casuística correspondeu às oportunidades de observação direta de 100 instalações e 100 desligamentos de hemodiálises (Hds), realizadas 
exclusivamente por auxiliares de enfermagem $(A E)$, nos meses de junho/julho de 2012, totalizando 23 dias.

Duas enfermeiras, com experiência clínica no processo de $\mathrm{Hd}$, realizaram as observações e documentaram o consumo de materiais, de soluções/medicamentos, a quantidade de AE envolvidos e o tempo despendido em cada instalação e desligamento de Hds, em instrumento desenvolvido com essa finalidade.

Aferiram-se os custos por meio da utilização dos custos diretos, dispêndio monetário que se aplica na produção de um produto ou de um serviço em que há possibilidade de identificação com o produto ou departamento. Custo direto é todo aquele que pode ser identificado e claramente quantificado ${ }^{(8)}$. Nas unidades hospitalares compõe-se basicamente de mão de obra, insumos e equipamentos utilizados diretamente no processo assistencial $^{(9)}$.

A mão de obra direta (MOD) refere-se ao pessoal que trabalha diretamente sobre um produto ou serviço prestado, desde que seja possível mensurar o tempo despendido e a identificação de quem executou o trabalho. Compõe-se dos salários, encargos sociais, provisões para férias e $13^{\circ}$ salário $^{(8)}$.

O Assistente Técnico IV, Recursos Humanos - Gestão de Pessoas do HC-FMUSP forneceu o salário médio de $\mathrm{R} \$$ 2.399,86 referentes à categoria AE. Como a jornada de trabalho da maioria desses profissionais, na sala de $\mathrm{Hd}$, corresponde a 30 horas semanais o custo da MOD por minuto foi de $R \$ 0,33$.
Obteve-se 0 custo dos materiais, soluções/medicamentos no Sistema de Administração de Materiais do Hospital.

O CTM das instalações e desligamentos das Hds foi calculado multiplicando-se o tempo despendido pelos $A E$ pelo custo unitário da MOD somando-se ao custo dos materiais, soluções/medicamentos. Para a realização dos cálculos utilizou-se a moeda brasileira $(R \$)$.

\section{RESULTADOS}

As 200 observações realizadas compreenderam 42 pacientes em urgência dialítica, portadores de CVC, sendo 23 mulheres com idade variando de 19 a 87 anos (mediana: 55 anos e moda 62 anos) e 19 homens com idade entre 27 e 81 anos (mediana: 62 anos e moda 80 anos). O número de sessões de Hd variou de uma a oito, sendo mais frequente a realização de uma sessão por paciente.

Todas as instalações e desligamentos de Hds foram executados por dois $A E$. A idade dos nove $A E$ integrantes do estudo variou de 23 a 60 anos (média de 41,9 anos); o tempo de atuação no IC-HCFMUSP de dois a 17 anos (média de 11 anos) e todos possuíam capacitação adequada à realização destes procedimentos.

A seguir mostrar-se-ão os valores descritivos da duração da instalação e desligamento de $\mathrm{Hds}$, em minutos, e seu CTM compreendendo custo com pessoal, com material e com soluções/medicamentos.

A partir dos dados da Tabela 1 observa-se que a duração da instalação variou de 4,00 a 90,00 minutos, com média de $8,60(D P \pm 10,20)$ e moda de 5,00 minutos.

Tabela 1: Distribuição da instalação das Hds segundo a duração, custo com pessoal, com material e com soluções/medicamentos. São Paulo, SP, Brasil, 2012.

\begin{tabular}{cccccccc}
\hline Variável & n & Média & DP & Mediana & Mínimo & Máximo & Moda \\
\hline Duração (em minutos) & 100 & 8,6 & 10,2 & 6 & 4 & 90 & 5 \\
Custo com pessoal & 100 & 5,64 & 6,73 & 3,96 & 2,64 & 59,4 & 3,3 \\
Custo com material & 100 & 47,68 & 0,83 & 47,76 & 46,41 & 51 & 47,76 \\
Custo com soluções/medicamentos & 100 & 26,78 & 18,39 & 20,93 & 20,54 & 143,14 & 20,92 \\
Custo total (em R\$) & 100 & 80,1 & 21,49 & 72,93 & 70,33 & 211,97 & 71,98 \\
\hline
\end{tabular}

O custo com pessoal variou de $R \$ 2,64$ a $R \$ 59,40$, com média de $R \$ 5,64$ ( $D P \pm 6,73$ ) e moda de $R \$ 3,30$. Quanto ao custo de material houve variação de $R \$ 46,41$ a $R \$ 51,00$, com média de $R \$ 47,68(D P \pm 0,83$ ) e moda de $\mathrm{R} \$ 47,76$.

Em relação ao custo com soluções e medicamentos houve variação de $R \$ 20,54$ a $R \$ 143,14$, com média de $R \$ 26,78(D P \pm 18,39)$ e moda de $R \$ 20,92$.

Os materiais e soluções/medicamentos que mais contribuíram para a elevação do custo das 100 instalações foram: linha arterial (100 unidades - R\$ $377,00)$; dialisador capilar de baixa eficiência $1,5 \mathrm{~m} 2$ (100 unidades: $\mathrm{R} \$ 3.615,00)$; linha venosa (100 unidades: $\mathrm{R} \$ 347,00)$; bicarbonato de sódio $8,4 \% \mathrm{com}$ água para injeção (100 galões de cinco litros: R\$ 800,00); solução polietrolítica (100 galões de cinco litros: $R \$ 1.038,00)$ e ativador tissular plasminogênio humano $1 \mathrm{mg} / \mathrm{ml}$ - 50ml (4 ml: R\$122,60).

O CTM acumulado referente às 100 instalações observadas foi de $\mathrm{R} \$ 8.010,00$ (100\%), sendo $\mathrm{R} \$ 564,0$ 
(7,04\%) com pessoal, $\mathrm{R} \$ 4.768,00$ (59,52\%) com material e R\$ $2.678,00 \quad(33,43 \%)$ com soluções/medicamentos.
Na Tabela 2 verifica-se a duração do desligamento variando de 4,00 a 10,00 minutos, com média de 6,30 $(D P \pm 1,64)$ e moda de 5,00 minutos.

Tabela 2: Distribuição do desligamento das Hds segundo a duração, custo com pessoal, com material e com soluções/medicamentos. São Paulo, SP, Brasil, 2012.

\begin{tabular}{cccccccc}
\hline Variável & n & Média & DP & Mediana & Mínimo & Máximo & Moda \\
\hline Duração (em minutos) & 100 & 6,3 & 1,64 & 5 & 4 & 10 & 5 \\
Custo com pessoal & 100 & 3,98 & 1,08 & 3,3 & 2,64 & 6,6 & 3,3 \\
Custo com material & 100 & 3,27 & 0,49 & 3,08 & 2,9 & 5,91 & 3,01 \\
Custo com soluções/medicamentos & 100 & 5,79 & 17,07 & 3,23 & 0,89 & 125,83 & 3,23 \\
Custo total (em R $\$$ ) & 100 & 13,04 & 17,01 & 10,16 & 7,04 & 132,16 & 9,38 \\
\hline
\end{tabular}

O custo com pessoal variou de $R \$ 2,64$ a $R \$ 6,60$, com média de $R \$ 3,98$ (DP $\pm 1,08$ ) e moda de $R \$ 3,30$. Em relação ao custo de material houve variação de $R \$$ 2,90 a R\$5,91, com média de R\$3, 27 (DP士 0,49) e moda de $R \$ 3,01$.

Quanto ao custo com soluções/medicamentos houve variação de $R \$ 0,89$ a $R \$ 125,83$, com média de $R \$ 5,79$ $(D P \pm 17,07)$ e moda de $R \$ 3,23$.

No momento do desligamento o ativador tissular plasminogênio humano $1 \mathrm{mg} / \mathrm{ml}$ - $50 \mathrm{ml}$ (8ml: $\mathrm{R} \$$ 245,20 ), seguido de heparina 5000UI/ml (311ml: $R \$$ $242,58)$ foram os medicamentos mais importantes para a composição do custo obtido.

O CTM acumulado referente aos 100 desligamentos observados foi de $R \$ 1.304,00$ (100\%), sendo $R \$ 398,00$ $(30,52 \%)$ com pessoal, $\mathrm{R} \$ 327,00$ (25,08\%) com material e R\$ 579,00 (44,40\%) com soluções e medicamentos.

Logo, o CTM da instalação e desligamento de Hds correspondeu a R\$93,14.

\section{DISCUSSÃO}

Os hospitais universitários são considerados centros de referência e de alta tecnologia e contribuem para o progresso técnico em saúde, especialmente nos países em desenvolvimento ${ }^{(10)}$. Sua estrutura deve oferecer serviços de alta tecnologia, apresentar eficiência no uso dos recursos e precisam, minimamente, conhecer os custos dos procedimentos que realizam ${ }^{(11)}$.

Em relação aos recursos humanos da sala de $\mathrm{Hd}$, o quadro de profissionais está em conformidade com a Resolução da Diretoria Colegiada-RDC no $154^{(12)}$. Destaca-se que todo profissional de enfermagem recémadmitido é acolhido por um enfermeiro responsável pelo processo de capacitação teórico-prática visando o alcance gradativo de melhores níveis de atuação junto aos pacientes submetidos à $\mathrm{Hd}$.

Como a execução da $\mathrm{Hd}$ requer dos profissionais constante observação dos sinais e sintomas apresentados pelo paciente, do funcionamento dos materiais e equipamentos utilizados e competência para tomar decisões adequadas à resolução de ocorrências ou para a minimização de suas consequências o investimento em educação continuada é imprescindível ${ }^{(4)}$.

Concorda-se com autores que afirmam que a educação continuada é fundamental para a tomada de consciência da necessidade da formação profissional sistemática, programada de modo a atingir todos os níveis, oferecendo a todos a oportunidade de crescimento profissional e pessoal ${ }^{(13)}$.

Os valores mais frequentes das idades dos pacientes observados indicam o predomínio de pessoas idosas. $\mathrm{Na}$ perspectiva do envelhecimento populacional, evidenciase que os idosos são grandes usuários dos serviços de saúde ${ }^{(14)}$ e que vem ocorrendo um aumento no consumo dos serviços por parte desse grupo populacional nos últimos anos ${ }^{(15)}$.

Assim, há a necessidade da adoção de estratégias que reduzam os custos sem prejuízos a qualidade da assistência prestada. Nesse prisma, os enfermeiros encontram-se em posição privilegiada para realização do gerenciamento de custos visto sua familiarização com a temática desde a formação acadêmica e o domínio de conhecimento específico da assistência em enfermagem ${ }^{(9)}$.

Ressalta-se que, se por um lado os profissionais de enfermagem prestam cuidados que consomem recursos materiais e financeiros, por outro lado geram resultados que concorrem à qualidade assistencial e aumentam o faturamento das organizações hospitalares. Então, os enfermeiros, ao decidirem as prioridades de seus serviços e quais recursos serão empregados em sua realização, representam relevante nível decisório na alocação dos mesmos ${ }^{(16)}$.

No contexto do estudo de caso único em questão, a maioria das instalações e desligamentos de $\mathrm{Hd}$ correspondeu à pacientes portadores de CVC de curta 
permanência inseridos em veia jugular interna direita, conforme recomendado na literatura ${ }^{(17)}$. Esses cateteres são inseridos por punção direta do vaso provendo acesso imediato a circulação sanguínea para viabilizar a execução da Hd. Requerem cuidados específicos em seu manuseio a fim de não torná-los fontes expressivas de infecção, complicação mais frequente e grave relacionada ao seu uso ${ }^{(18)}$.

Durante a coleta de dados os enfermeiros não participaram da instalação e desligamento de nenhuma sessão de Hd. Isso porque dedicam maior parte do seu tempo às intervenções de enfermagem de cuidado indireto, compreendendo o processo de trabalho na dimensão do gerenciamento do cuidado e da equipe de enfermagem, enquanto que os profissionais de enfermagem de nível médio dedicam-se às intervenções de cuidado direto, isto é, ao processo de trabalho assistencial.

Enfatiza-se que na sala de $\mathrm{Hd}$ os procedimentos estudados constituem-se em cuidados diretos que podem ser definidos como tratamentos realizados por meio da interação com o paciente, configurando-se nas ações de enfermagem de aspecto fisiológico abarcando ações práticas e de apoio ${ }^{(19)}$.

Os materiais componentes do circuito extracorpóreo e os galões de bicarbonato de sódio $8,4 \%$ e os de solução polieletrolítica foram os itens que mais contribuíram para a elevação do custo da instalação.

$O$ ativador tissular plasminogênio humano, antitrombolítico empregado nos casos de desobstrução de CVC, foi o medicamento que contribuiu significativamente para a composição do CTM no momento da instalação, apesar da pouca quantidade utilizada $(4 \mathrm{ml})$. No desligamento este mesmo medicamento, seguido da heparina $5000 \mathrm{UI} / \mathrm{ml}$ foram os mais importantes para a composição do CTM.

Salienta-se que os materiais, soluções/medicamentos estudados são adquiridos pelo Hospital por meio de uma modalidade de licitação utilizada no Brasil denominada Pregão. Ela é considerada como um aperfeiçoamento do regime de licitações para a Administração Pública Federal, Estadual, Distrital e Municipal, uma vez que possibilita o incremento da competitividade e ampliação das oportunidades de participação nas licitações, por parte dos licitantes Pessoas Jurídicas ou Pessoas Físicas interessadas em vender bens e/ou serviços comuns conforme os editais e contratos que visam o interesse público ${ }^{(20)}$.

Não foram objeto de observação outras atividades de cuidados diretos, tais como, verificação dos sinais vitais, pesagem, controle de glicemia capilar, auxílio para alimentação, atendimento de ocorrências com o paciente, com os materiais e/ou com os equipamentos, bem como administração de medicamentos, utilizados durante as sessões de $\mathrm{Hd}$.

Também não foram focalizadas as intervenções de cuidados indiretos, ou seja, aquelas que compreendem um tratamento realizado longe do paciente, mas, em seu benefício abrangendo ações voltadas para o gerenciamento da unidade e de colaboração interdisciplinar ${ }^{(19)}$. Como exemplos desse tipo de cuidado citam-se o preparo do circuito extracorpóreo e sua montagem na máquina de $\mathrm{Hd}$; a documentação das ocorrências, das ações desenvolvidas e da evolução do paciente a cada sessão; a limpeza e desinfecção de mobiliários e equipamentos, entre outros.

Diante do CTM $(R \$ 93,14)$ relativo a instalação e ao desligamento da $\mathrm{Hd}$, considerando que foram custeadas duas das principais intervenções de cuidados diretos e não foi aferido o custo de nenhuma intervenção de cuidado indireto, é possível inferir que o repasse monetário de $\mathrm{R} \$ 111,42$ previsto no $\operatorname{SIGTAP}^{(6)}$ ao IC do HCFMUSP, referentes a cada sessão de $\mathrm{Hd}$ para pacientes renais agudos/crônicos agudizados sem tratamento dialítico iniciado, é insuficiente para cobrir o investimento financeiro.

Os hospitais da rede pública vêm enfrentando dificuldades para gerirem seus recursos escassos em consequência da diminuição dos gastos federais, estaduais e municipais com saúde frente ao aumento das demandas da população por serviços de saúde ${ }^{(9)}$.

A limitação de recursos torna necessário o conhecimento dos custos da assistência prestada visando o gerenciamento dos custos dos serviços hospitalares, pois contribui com as tomadas de decisão quanto à alocação de recursos e investimentos futuros, bem como para comparar e analisar os valores pagos pelo SUS demonstrando, assim, a premência de melhores repasses de verba ${ }^{(3)}$.

Os profissionais de saúde precisam ter clareza de que a prestação de cuidados, em diferentes contextos assistenciais, gera custos, diretos e indiretos, e o seu conhecimento desencadeará reflexões e discussões quanto ao uso criterioso e adequado dos recursos humanos e materiais disponíveis ${ }^{(21)}$.

Nessa direção, acredita-se que o presente estudo representa uma abordagem inicial para a identificação dos custos diretos inerentes ao processo de $\mathrm{Hd}$ a fim de assegurar a eficiência alocativa dos recursos humanos, materiais, estruturais e econômicos envolvidos nesta 
modalidade de TRS predominante para os portadores de DR.

\section{CONCLUSÃO}

$\mathrm{Na}$ área da saúde constata-se a elevação vertiginosa dos custos para o atendimento das diferentes demandas de cuidados, sendo essencial a realização de estudos sobre os aspectos financeiros envolvidos.

Tais estudos possibilitam a elaboração de estratégias para o uso racional dos recursos, equilibrando a oferta de serviços de saúde e a viabilidade dos custos. A nível local a identificação do CTM da instalação e do desligamento da Hd suscitará a análise crítica do repasse monetário do SUS ao IC-HCFMUSP e contribuirá, especificamente, para desencadear reflexões acerca do gerenciamento de custos na sala de Hd.

Os resultados evidenciados poderão, ainda, estimular o desenvolvimento de novas investigações, abrangendo todas as intervenções de cuidados diretos e as de cuidados indiretos, que auxiliarão aos coordenadores e profissionais de enfermagem a detectar ineficiências, desperdícios e intervir no processo produtivo colaborando, efetivamente, com os gestores hospitalares no gerenciamento eficiente dos recursos disponíveis utilizados nos Serviços de Hd.

13. Ferreira JCOA, Kurcgant P. Capacitação profissional do enfermeiro de um complexo hospitalar de ensino na visão de seus gestores. Acta paul. enferm. [Internet]. 2009 [acesso em: 31 dez 2014];22(1):31-6. Disponível em: http://dx.doi.org/10.1590/S0103-21002009000100005. 14. Lima-Costa MF, Loyola Filho AI, Matos DL. Tendências nas condições de saúde e uso de serviços de saúde entre idosos brasileiros: um estudo baseado na Pesquisa Nacional por Amostra de Domicílios (1998, 2003). Cad Saude Publica [Internet]. 2007 [acesso em: $31 \mathrm{dez} 2014$ ];23(10):2467-78. Disponível em: http://dx.doi.org/10.1590/S0102-

311X2007001000021.

15. Veras R. Population aging today: demands, challenges and innovations. Rev Saude Publica [Internet]. 2009 [acesso em: 31 dez 2014];43(3):548-54. Disponível em:

http://dx.doi.org/10.1590/S0034-89102009005000025. 16. Zunta RSB, Castilho V. Billing of nursing procedures at an intensive care unit. Rev Lat Am Enfermagem [Internet]. 2011 [acesso em: $31 \mathrm{dez} 2014] ; 19(3)$ : 573-80. Disponível em: http://dx.doi.org/10.1590/S0104-11692011000300017. 17. Allon M, Work J. Venous Catheter Access for Hemodialysis. In: Daugirdas JT, Blake PG, Ing TS. Handbook of Dialysis. $4^{\text {th }}$ ed. Philadelphia: Lippincott Williams \& Wilkins; 2007. p. 87-104. 18. Souza RA, Oliveira EA, Silva JMP, Lima EM. Avaliação do acesso vascular para hemodiálise em crianças e adolescentes: um estudo de coorte retrospectivo de 10 anos. J Bras Nefrol [Internet]. 2011 [acesso em: $31 \mathrm{dez} 2014$ ];33(4):422-30. Disponível em:

http://www.scielo.br/scielo.php?script=sci_arttext\&pid=S010128002011000400006.

19. Bulechek GM, Butcher HK, Dochterman JMM, Wagner C. Nursing Interventions Classification (NIC). $6^{\text {th }}$ ed. St. Louis: Mosby; 2012.

20. Lei no 10.520, de 17 de julho de 2002 (BR) [Internet]. Institui, no âmbito da União, Estados, Distrito Federal e Municípios, nos termos do art. 37, inciso XXI, da Constituição Federal, modalidade de licitação denominada pregão, para aquisição de bens e serviços comuns, e dá outras providências. Diário Oficial da União. 18 jul 2002 [acesso em: 31 dez 2014]. Disponível em:

http://www.planalto.gov.br/ccivil_03/leis/2002/l10520.htm. 21. Lima AFC, Castilho V, Fugulin FMT, Silva B, Ramin NS, Melo TO. Costs of most frequent nursing activities in highly dependent hospitalized patients. Rev Lat Am Enfermagem [Internet]. 2012 [acesso em: $31 \mathrm{dez} 2014] ; 20(5): 880-7$. Disponível em: http://dx.doi.org/10.1590/S0104-11692012000500009.

Artigo recebido em 12/03/2013.

Aprovado para publicação em 30/06/2014.

Artigo publicado em 31/12/2014. 2004. Estabelece o Regulamento Técnico para o funcionamento dos Serviços de Diálise. Diário Oficial da União. 17 jun 2004. 